\title{
Effetti della lettura ad alta voce oltre il linguaggio: l'autoregolazione come mediatore
}

\author{
Piccolo LDR, Weisleder A, Oliveira JBA, et al. \\ Reading Aloud, Self-Regulation, and Early Language and Cognitive Development in Northern Brazil \\ J Dev Behav Pediatr. 2021 Jul 1. doi: 10.1097/DBP.0000000000000985. Epub ahead of print
}

\author{
Rubrica L'articolodelmese \\ a cura di Costantino Panza
}

La lettura ad alta voce è un'attività familiare che se attuata con frequenza nei primi anni di vita sostiene lo sviluppo linguistico e l'alfabetizzazione del bambino. Essendo un processo sociale, la lettura condivisa in famiglia migliora lo sviluppo psicosociale del bambino e le competenze genitoriali. Recentemente è stato dimostrato che la stimolazione cognitiva e linguistica sono associate a un miglioramento delle abilità di autoregolazione. Questo articolo descrive le più recenti acquisizioni sull'importanza della conversazione durante la lettura condivisa e la relazione di questa attività con l'autoregolazione e la funzione genitoriale.

Effects of reading aloud beyond language: self-regulation as a mediator

Reading aloud is a family activity which, if carried out frequently in the first years of life, supports the child's linguistic and alphabetic development. Being a social process, shared reading in the family improves the child's psychosocial development and parenting skills. It has recently been shown that cognitive and linguistic stimulation are associated with an improvement in self-regulation skills. This article describes the most recent findings on the importance of conversation during shared reading and the relationship of this activity with self-regulation and parenting.

\section{Background}

La lettura ad alta voce o la lettura condivisa è un'attività che può migliorare lo sviluppo del linguaggio dei bambini nei primi anni di vita. La lettura del libro è un' ottima stimolazione cognitiva, migliora lo sviluppo socioemozionale e riduce le disparità in famiglie a basso reddito o con genitori con scarsa scolarità. Ad oggi non ci sono studi che indichino l'influenza di questa attività tra genitore e bambino nei primi anni di vita sull'autoregolazione del bambino.

\section{Scopi}

Valutare se il programma di lettura ad alta voce (Reach Out and Read) realizzato in una comunità in Brasile migliora lo sviluppo dell'autoregolazione del bambino; inoltre valutare se gli estiti cognitivi sono mediati dall'autoregolazione.

\section{Metodi}

RCT a cluster realizzato a Boa Vista (Brasile) in comunità a basso reddito; l'arruolamento dei bambini di 2-4 anni è avventuto tra quelli frequentanti asili o scuole dell'infanzia (484 bambini). Nel gruppo di intervento (232 bambini) i genitori hanno frequentato 9 workshop (un incontro ogni tre settimane condotto da psicologo con training specifico) con discussione sulle strategie di lettura ad alta voce, sulla genitorialità positiva, sulle esperienze di gioco e lettura con i bambini; inoltre le famiglie hanno avuto accesso a un prestito di libri adatti all'età dei bambini presso la biblioteca locale coinvolta nel programma di lettura.

\section{Risultati}

I bambini del gruppo di intervento hanno mostrato un'autoregolazione significativamente più alta $(\mathrm{d}$ di Cohen $=0.25)$ rispetto al gruppo di controllo, in particolare nei sottodomini dell' attenzione $(\mathrm{d}=0.24)$ e del controllo inibitorio $(\mathrm{d}=0.21)$. L'impatto sul vocabolario ricettivo, sul QI e sulla memoria di lavoro risultano mediati dall'autoregolazione. Gli effetti della lettura ad alta voce sul linguaggio e le capacità cognitive sono spiegati attraverso la stimolazione cognitiva in famiglia, la lettura con conversazione, caratterizzata da un'interazione genitoriale ricca di responsività verbale, stile affettuoso e sensitività, e l'autoregolazione.

\section{Conclusioni}

L'autoregolazione rappresenta un importante meccanismo attraverso il quale gli interventi di lettura ad alta voce influenzano il linguaggio e gli esiti cognitivi.

\section{Commento}

Cinquant'anni fa si consigliava di evitare di utilizzare i libri con i bambini piccoli perchè la lettura era considerata un'attività che poteva danneggiare il normale sviluppo del bambino di età prescolare. Altri tempi - non tanto lontani - e convinzioni che sono state ribaltate dallosservazione e da numerosi studi scientifici.

\section{Lettura e alfabetizzazione prima dei 6 anni di età}

Lintervento di lettura ad alta voce più conosciuto in ambito delle cure primarie, Reach Out and Read, è stato oggetto di molte pubblicazioni scientifiche che hanno confermato il successo delle guide anticipatorie del pediatra e del dono di libri alle famiglie sul miglioramento dell'alfabetizzazione del bambino; anche in Italia il programma Nati per Leggere $(\mathrm{NpL})$ si è dimostrato efficace: in un trial svolto in Veneto la valutazione del linguaggio effettuata all'età di cinque anni ha evidenziato che $\mathrm{i}$ bambini esposti alla lettura condivisa almeno quattro volte la settimana avevano una ricchezza di vocabolario significativamente superiore ai bambini 
esposti occasionalmente o non esposti a NpL [1]. Infine, la più recente metanalisi sull'efficacia della lettura condivisa sull'alfabetizzazione, effettuata su soli RCT (19 studi, 2.594 partecipanti, bambini di età dai 8 a 66 mesi), ha rilevato un piccolo effetto sul linguaggio espressivo $(\mathrm{d}$ di Cohen $=0.41)$ e recettivo $(\mathrm{d}=0.26)$ con un grande effetto sulla competenza del genitore alla lettura condivisa $(\mathrm{d}=1.01)$, una qualità specifica che indica la capacità ad applicare lo stile di lettura con conversazione [2]; in tutti questi studi il genitore o il caregiver ha parteciapto a un training di 1 o più sedute (fino a 18) della durata di 15-120 minuti/sessione per apprendere lo stile di lettura con conversazione.

\section{Dall'alfabetizzazione agli effetti psicosociali}

Ma gli effetti della lettura ad alta voce in famiglia non si fermano all'alfabetizzazione. Una recente metanalisi [3] ha raccolto 19 trial (3.264 famiglie con bambini di 0-6 anni) sulla lettura ad alta voce tra genitore e bambino valutandone gli effetti psicosociali; gli interventi sono stati effettuati nelle cure primarie o presso agenzie educative, biblioteche o a domicilio e, nella maggior parte dei casi, la popolazione arruolata era a basso reddito o con scarsa scolarità materna o con bambini con ritardo di linguaggio o in situazioni di svantaggio sociale. Gli esiti misurati sul bambino (regolazione socio-emotiva, problemi di comportamento, qualità di vita, interesse alla lettura) e sul genitore (stress/depressione, competenza, relazione con il figlio, attitudine alla lettura ad alta voce) hanno rilevato effetti positivi anche se di piccola entità e di cui abbiamo parlato estesamente in un precedente Articolo del mese. Anche in tutti i trial contenuti in questa revisione $\mathrm{i}$ genitori hanno avuto un training di gruppo o attraverso incontri individuali che variavano da 2 fino a 28 sessioni con volontari addestrati (la maggior parte) o con professionisti.

Questi effetti della lettura in famiglia non ci devono stupire: il condividere la lettura di un libro tra un adulto ed un bambino è molto più che un semplice trasferimento di informazioni: in questo processo sociale non è presente un semplice scambio di parole o gesti, ma è presente una dimensione affettiva che definisce la relazione tra genitore e bambino; la lettura di un libro è un vero e proprio atto sociale legato alla relazione affettiva [4]. Ed è qui che entra in gioco l'autoregolazione.

\section{Cos'è l'autoregolazione}

La regolazione è un processo biologico e può essere definita come la capacità che un bambino possiede, fin dalla nascita, di regolare i propri stati emotivi e organizzare l'esperienza e le risposte comportamentali [5]. L'emergere delle abilità di autoregolazione è una componente essenziale dello sviluppo e rappresenta nei primi anni della crescita un risultato di notevole complessità: le funzioni esecutive e la capacità di regolare le emozioni, abilità necessarie per raggiungere i propri obiettivi, sono mediate dalla capacità individuale di regolazione [6]. L'autoregolazione comprende una vasta gamma di processi come la regolazione fisiologica (ad esempio fame, veglia, sonno e pianto nel lattante), la regolazione delle emozioni, il controllo volontario, il controllo della fatica, l'autocontrollo, il controllo inibitorio, la capacità esecutiva. Il controllo volontario del comportamento e delle emozioni si sviluppa soprattutto nel secondo anno di vita e viene considerato un traguardo evolutivo fondamentale per le successive abilità in campo sociale del bambino [7]. Inizialmente i bambini necessitano della presenza dei genitori o caregiver che sostengano il processo di regolazione offrendo strategie di regolazione che successivamente saranno interiorizzate dal bambino: la co-regolazione descrive un adattamento reciproco genitore-bambino che si svolge continuamente durante tutto il processo di interazione ed è presente già dai primi mesi di vita. Una mancata funzione regolativa del genitore può costringere il bambino a forme di regolazione improprie o autoconsolazione che possono alterare la traettoria dello sviluppo sociale e del comportamento. Sono descritte tre dimensioni della funzione genitoriale che favoriscono lo sviluppo delle funzioni esecutive e dell'autoregolazione: a) la sensitività che consiste nella capacità di osservare, interpretare e di rispondere adeguatamente ai segnali del bambino non solo dal punto di vista fisico, ma anche emotivo; b) lo scaffolding (termine inglese che significa impalcatura), ossia il sostegno che il genitore offre al bambino per aiutarlo a risolvere un problema o a raggiungere un obiettivo, consapevole che il bambino non riuscirebbe a raggiungere quell'obiettivo senza un adeguato sostegno e quindi propone strategie riconoscendo le possibilità di comprensione del bambino in quel dato momento dello sviluppo; c) la mind mindedness, una competenza mentale specifica del genitore che attribuisce al bambino stati mentali e intenzioni fin dalle sue prime fasi di vita e che si rivela attraverso la tendenza del genitore ad utilizzare termini mentali mentre parla al bambino, riconoscendo in lui non un essere con semplici bisogni da soddisfare, ma un individuo dotato di una mente e che pertanto ha desideri, credenze, aspettative, obiettivi e sentimenti, il genitore con questa competenza non si ferma a descrivere il bambino semplicemente nei suoi attributi fisici o in base al suo comportamento [8]. Queste tre caratteristiche della funzione genitoriale rappresentano i modi con cui il caregiver può agire come regolatore esterno, sostenendo gradualmente le competenze di autoregolazione del bambino stesso. In questi momenti, se i libri fanno parte della vita del bambino, risultano essere uno strumento strategico per il genitore.

\section{La conversazione}

Il genitore o il caregiver ha un'ottima opportunità per offrire al bambino strategie di regolazione attraverso le continue interazioni che stabilisce con il bambino durante i momenti di relazione. Le complesse competenze sociali emergenti nelle prime fasi di sviluppo e le competenze comunicative e di regolazione emotiva del bambino sono favorite dall'intersoggettività, una capacità innata che si manifesta come immediata e naturale consapevolezza della presenza dell'altro [9]; questi momenti di interazione, presenti già nei primi mesi di vita sono chiamati, non a caso, protoconversazioni nelle quali il bambino, attratto dalla voce, dall' espressione del volto, dai gesti delle mani, risponde giocosamente imitando e provocando imitazione; il genitore nelle protoconversazioni guarda e ascolta, anticipando intuitivamente le espressioni del bambino, e risponde empaticamente e giocosamente con linguaggio motherese, toccando ed esprimendo con il corpo, le mani e il volto il proprio stato emotivo (Figura 1).

Diversi recenti studi hanno evidenziato che il bambino non solo è in grado di fare conversazione, ma che desidera conversare (turn taking) con il genitore attraverso vocalizzazioni primarie definite protofoni (protophones in inglese, ad esempio pronuncia di vocali, consonanti, strilli, sussurri) già a partire dai primi giorni di vita [10]. Ecco alcuni recenti contributi scientifici 
Figura 1. In una protoconversazione la mamma e il bambino comunicano attraverso diverse modalità di percezione e di espressione trasmettendo informazioni ed emozioni attraverso i gesti, lo sguardo, la voce, l'espressione facciale (da voce bibliografica 14).

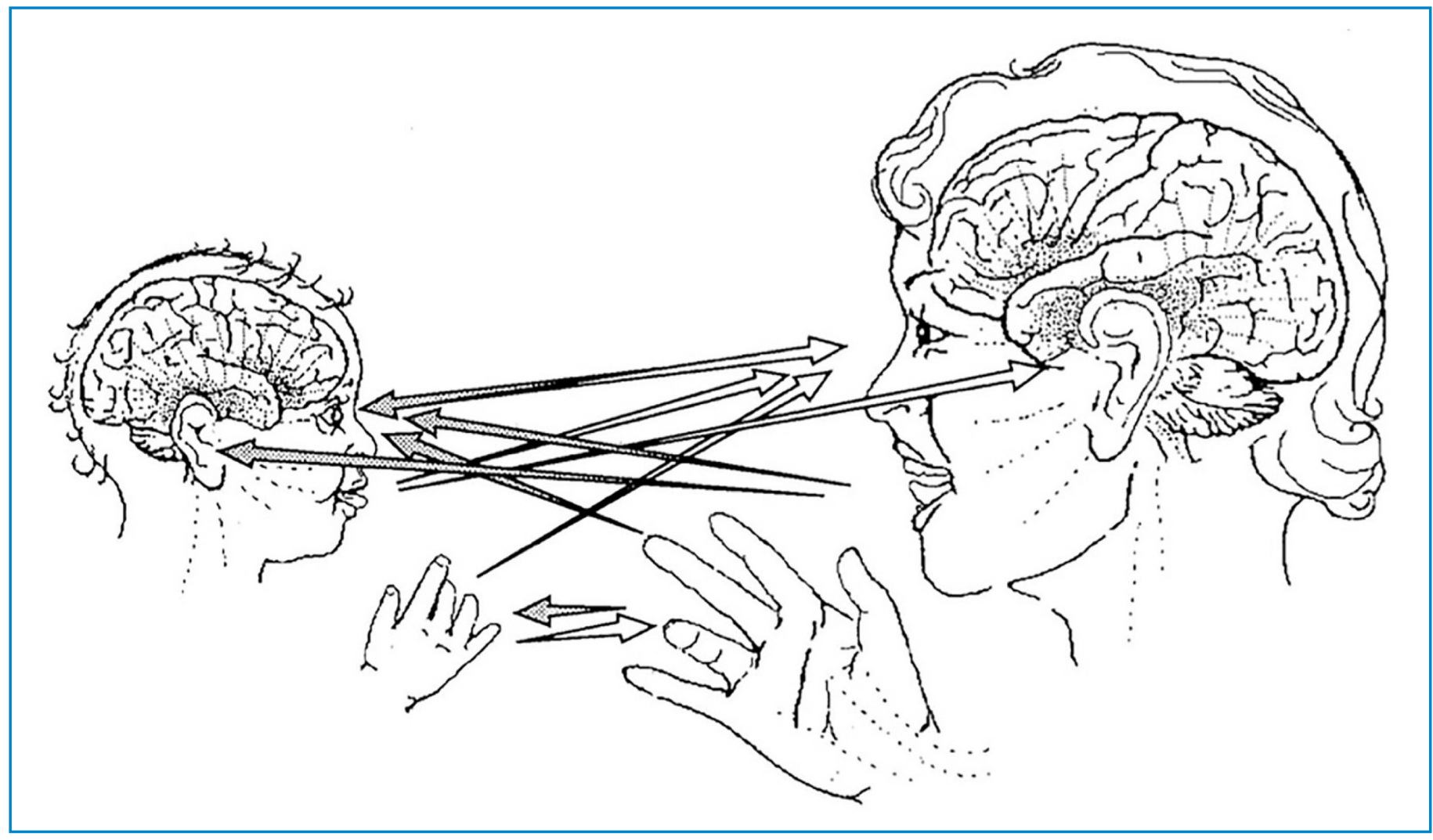

sull'importanza della conversazione tra genitore e bambino nella prima infanzia: neonati pretemine di 32-36 settimane di età gestazionale ricoverati in terapia intensiva neonatale vocalizzano seguendo il turn taking con il genitore in misura direttamente proporzionale alle parole rivolte loro dall'adulto e questo aumento di turn taking in età neonatale è associato a un miglioramento dello sviluppo del linguaggio a 18 mesi [11]; un aumento di turn taking all' età di 5-8 mesi è associato a una precoce maturazione di due reti cerebrali di connessione coinvolte nella comprensione del linguaggio [12]; i turni di conversazione genitore-bambino nei primi due anni di vita (soprattutto a 18-24 mesi) sono associati a un miglioramento del QI, della comprensione verbale, del linguaggio espressivo e recettivo misurati a 9-14 anni di età [13]. In questa visione dello sviluppo del bambino, il libro può essere considerato un formidabile strumento per favorire l'interazione verbale e affettiva con il genitore attraverso, appunto, la conversazione.

\section{L'albo illustrato "tradizionale" come strumento per conversare} Uno studio osservazionale condotto su 26 diadi madre-bambino di 10-16 mesi di età ha osservato la qualità della comunicazione tra genitore e figlio durante 15 minuti di interazione svolta con tre tipi di giochi: giocattoli tradizionali, giochi elettronici che producevano luci, parole, canti e frasi, e libri illustrati. Nel confronto tra i diversi tipi di gioco, la diade impegnata nella lettura del libro ha presentato un maggior numero di parole pronunciate dal genitore associato ad un più ricco vocabolario, maggiori vocalizzazioni del bambino, un più elevato numero di turni di conversazione della diade e di risposte del genitore [14]. Un'altra ricerca ha confrontato la modalità di relazione tra genitore e figlio (24-36 mesi) durante la lettura di un libro elettronico, sia interattivo che non interattivo, su tablet e un albo illustrato cartaceo; i ricercatori hanno valutato l'interazione tra genitore e bambino misurando la lettura dialogica, la lettura non dialogica, le verbalizzazioni riguardanti il supporto cartaceo o tablet, le verbalizzazioni negative (ad esempio: "non toccare qui", "lascia stare il pulsante"), e le interazioni non verbali come la condivisione degli stati emotivi e l'esperienza di collaborazione e di influenza reciproca durante la lettura. La più elevata interazione verbale positiva del genitore e del bambino è avvenuta in occasione della lettura dell'albo illustrato cartaceo, mentre i risultati peggiori sono stati ottenuti dalla lettura del libro elettronico interattivo, nel quale la conversazione era disturbata ad esempio dal tocco sull'oggetto o sul personaggio che produceva un rumore o l'audio della parola corrispondente, oppure un suono ambientale riferito alla scena rappresentata a ogni apertura di pagina. La condivisione degli stati emotivi era simile in tutte e tre le esperienze di lettura mentre l'atteggiamento di reciproca collaborazione era massima durante la lettura del libro cartaceo; infine, durante la lettura dell'e-book era massimo il comportamento di controllo sociale ed era presente un orientamento corporeo più solitario dei bambini, indicatore di scarsa esperienza di condivisione [15]. Il libro illustrato cartaceo, rispetto ad altri formati elettronici o altri giochi, ha le peculiarità di offrire la migliore esperienza di condivisione e questo è stato dimostrato in un trial sul campo svolto in Sudafrica presso famiglie svantaggiate: i genitori che avevano avuto un training sulla lettura condivisa miglioravano la propria interattività verso i bambini in termini di sensitività (seguivano con attenzione l'interessa del bambino e offrivano risposte contingenti), di elaborazione (conversazione 
sulle immagini del libro), di reciprocità (turni di conversazione, sguardo reciproco). Durante il gioco con oggetti, invece, l'interazione tra genitore e figlio presentava solo un leggero miglioramento nell'ambito della sensitività. I miglioramenti nel comportamento prosociale dei bambini così come negli esiti cognitivi erano mediati dall'interazione che si svolgeva durante la lettura ad alta voce e non dall'interazione durante il gioco con oggetti [16]. Ma le continue interazioni offerte dalla lettura condivisa, che sostengono la continua regolazione intersoggettiva della diade e che portano a sensibili miglioramenti nel comportamento prosociale del bambino agiscono anche sul genitore che riduce l'utilizzo di uno stile duro e punitivo [17].

\section{Il genitore ha necessità di apprendere lo stile di lettura con con- versazione}

Il punto cruciale tuttavia non è far sapere che leggere ad alta voce fa bene (sia al genitore che al bambino) ma aiutare il genitore $\mathrm{e}$ sostenerlo nel leggere e conversare con il bimbo. Molti genitori, soprattutto i meno scolarizzati, dialogano poco con i loro bambini preferendo frasi di dissuasione ("No", "Non toccare", "Non farlo", "Stai fermo") e utilizzano molto poco le frasi di incoraggiamento o di spiegazione [18]. I genitori delle famiglie a maggior rischio di povertà educativa hanno difficoltà a considerare la lettura come un'attività da svolgere con il bambino $[19,20]$ o non usano lo stile di lettura più adeguato rendendo l'esperienza della lettura non divertente e coinvolgente [21]; oggi, in realtà, in molte famiglie sono i media device che dialogano con i bambini piccoli. Le guide anticipatorie del pediatra o gli incontri con i volontari di NpL hanno lo scopo di favorire la familiarizzazione con l'oggetto libro, spiegare al genitore le tappe dello sviluppo in modo da non avere aspettative irrealistiche sulle capacità del bambino e indicare lo stile di lettura dialogico in modo da ingaggiare il bambino in una conversazione con il genitore.

Una revisione sistematica di interventi di sostegno ai genitori alla lettura ad alta voce ha rilevato che quando il genitore ha avuto un training per la lettura dialogica la misura dell'effetto sull'alfabetizzazione è alta $(\mathrm{d}=1.18)$, mentre se il genitore leggeva ad alta voce senza training l'efficacia è decisamente modesta $(\mathrm{d}=0.18)$ [22].

\section{Cosa può fare il pediatra}

Sappiamo che il successo di una attività familiare come la lettura condivisa può dipendere dalle risorse familiari. Il pediatra non può intervenire sull'abitazione (scarsi spazi, isolamento o degrado urbano, affollamento abitativo) ma può arricchire di libri la famiglia; infatti il successo della lettura è direttamente proporzionale al numero di albi illustrati presenti in casa [23]. Oltre il $10 \%$ delle famiglie con lattanti non ha alcun libro per bambini in casa, e oltre il $20 \%$ dei genitori non prevedono di leggere libri per bambini [20]. Rifornire le abitazioni di queste famiglie di tanti libri può essere un obiettivo da perseguire attraverso partnership con la locale biblioteca comunale per prestiti dedicati; il pediatra potrebbe promuovere un crowfunding o altro tipo di finanziamento per acquistare libri da donare alle famiglie più svantaggiate, oppure promuovere le attività proposte a livello gratuito nei Villaggi per crescere (Home - Villaggio per crescere) in modo da supplire alle carenze dell' abitazione con uno spazio dedicato alle famiglie dove far giocare il bambino offrendogli i migliori stimoli cognitivi. Oltre la guida anticipa- toria, che deve essere sempre presente a ogni bilancio di salute nei primi anni di vita, il pediatra potrebbe offrire occasioni di apprendimento della lettura condivisa a quei genitori che per stato socio economico, scolarità o particolari vulnerabilità familiari potrebbero essere in difficoltà a leggere secondo lo stile della lettura con conversazione. Gli incontri con questi genitori, in piccoli gruppi o individualmente dovrebbero essere condotti ad esempio dai volontari NpL presenti nella struttura ambulatoriale o nella biblioteca di riferimento: compito del pediatra è quello di partecipare all'attivazione dei corsi per volontari NpL e di prendere accordi con la locale biblioteca. Il supporto ai genitori è fondamentale nel primo anno di vita, quando il bambino approccia i libri e dimostra un interesse labile. È in questi momenti che la guida anticipatoria risulta cruciale, per rassicurare i genitori e invitarli a continuare a proporre i libri al bambino come un'attività ludica breve ma costante finalizzata a sviluppare un'affezione all'oggetto libro e a tale modalità relazionale. La lettura ad alta voce è un processo sociale che richiede competenze educative positive; se per la gran parte dei genitori queste funzioni genitoriali sono spontanee, non intenzionali, altri genitori hanno necessità di apprendere ed esercitarsi alla genitorialità positiva. Realizzare incontri o programmare dei corsi per genitori incentrati sulla genitorialità è un altro compito di un pediatra che esce dalla routine ambulatoriale e che entra nella rete territoriale dei servizi di sostegno alla famiglia. Infine ricordarsi che il genitore, oltre la mamma, vuol dire anche papà: sensitività, scaffolding e mentalizzazione sono caratteristiche sia materne che paterne. Interventi dedicati in modo specifico ai padri risultano essere efficaci sia sulle compentenze del genitore che sugli esiti dei bambini anche nella letttura condivisa [24]. Il compito del pediatra è quello di modificare i microsistemi che ruotano attorno al bambino in modo da sostenere le migliori opportunità di crescita ricordandosi che è l'ambiente - detto in altre parole il codice postale - a giocare un ruolo cruciale sul neurosviluppo (cognizione, funzione esecutiva, capacità attentiva, autoregolazione, sviluppo motorio, emotivo, di linguaggio, di comportamento) mentre solo il $10 \%$ dello sviluppo è sostenuto dal codice genetico [25].

1. Toffol G, Melloni M, Cagnin R, et al. Studio di efficacia del progetto "Nati per Leggere". Valutazione degli effetti della lettura ad alta voce da parte dei genitori sullo sviluppo del linguaggio dei bambini. Quaderni acp 2011; 18(5): 195-201

2. Dowdall N, Melendez-Torres GJ, Murray L, et al. Shared picture book reading interventions for child language development: A systematic review and meta-analysis. Child development. 2020;91(2):e383-99.

3. Xie QW, Chan CHY, Ji Q, et al. Psychosocial Effects of Parent-Child Book Reading Interventions: A Meta-analysis. Pediatrics. 2018;141(4):e20172675

4. Bus AG, van Ijzendoorn $\mathrm{MH}$. Affective dimension of mother-infant picturebook reading. Journal of School Psychology 1997; 35(1): 47-60 5. Simonelli A. La funzione genitoriale. 2014 Raffaello Cortina Editore 6. Buckner JC, Mezzacappa E, Beardslee WR. Self-regulation and its relations to adaptive functioning in low income youths. American Journal of Orthopsychiatry 2009;79(1):19.

7. Kim S, Kochanska G. Child temperament moderates effects of parent-child mutuality on self-regulation: a relationship-based path for emotionally negative infants. Child Dev. 2012;83(4):1275-89

8. Bernier A, Carlson SM, Whipple N. From external regulation to sel- 
f-regulation: early parenting precursors of young children's executive functioning. Child Dev. 2010;81(1):326-39

9. Trevarthen C, Aitken KJ. Infant intersubjectivity: Research, theory, and clinical applications. J Child Psychol Psychiatry. 2001;42(1):3-48

10. Oller DK, Caskey M, Yoo $H$, et al. Preterm and full term infant vocalization and the origin of language. Sci Rep. 2019;9(1):14734

11. Caskey M, Stephens B, Tucker R, et al. Adult talk in the NICU with preterm infants and developmental outcomes. Pediatrics. 2014;133(3):e578-84

12. King LS, Camacho MC, Montez DF, et al. Naturalistic Language Input is Associated with Resting-State Functional Connectivity in Infancy. J Neurosci. 2021;41(3):424-434

13. Gilkerson J, Richards JA, Warren SF, et al. Language Experience in the Second Year of Life and Language Outcomes in Late Childhood. Pediatrics. 2018;142(4). pii: e20174276

14. Sosa AV. Association of the Type of Toy Used During Play With the Quantity and Quality of Parent-Infant Communication. JAMA Pediatr. 2016;170(2):132-7

15. Munzer TG, Miller AL, Weeks HM, et al. Differences in parent-toddler interactions with electronic versus print books. Pediatrics. 2019;143(4):e20182012

16. Murray L, De Pascalis L, Tomlinson M, et al. Randomized controlled trial of a book-sharing intervention in a deprived South African com $\neg$ munity: effects on carer-infant interactions, and their relation to infant cognitive and socioemotional outcome. J Child Psychol Psychiatry. 2016;57(12):1370-1379

17. Jimenez ME, Mendelsohn AL, Lin Y, et al. Early Shared Reading Is Associated with Less Harsh Parenting. Journal of developmental \& behavioral pediatrics 2019;40(7):530-537

18. Hart B. Risley TR. Meaningful differences in the everyday experience of young American children. Paul H Brookes Publishing 1995.

19. El Moussaoui N, Braster S. Perceptions and practices of stimulating children's cognitive development among Moroccan immigrant mothers. Journal of child and family studies. 2011;20(3):370-83.

20. Berkule SB, Dreyer BP, Huberman HS, Fierman AH, Mendelsohn AL. Attitudes about shared reading among at-risk mothers of newborn babies. Ambulatory Pediatrics. 2007;7(1):45-50.

21. Weigel DJ, Lowman JL, Martin SS. Language development in the years before school: A comparison of developmental assets in home and child care settings. Early Child Development and Care. 2007;177(67):719-34.

22. Lonigan CJ, Shanahan T. Developing Early Literacy: Report of the National Early Literacy Panel. Executive Summary. A Scientific Synthesis of Early Literacy Development and Implications for Intervention. National Institute for Literacy. 2009

23. Szumlas GA, Petronio P, Mitchell MJ, et al. A Combined Reach Out and Read and Imagination Library Program on Kindergarten Readiness. Pediatrics. 2021;147(6):e2020027581.

24. Chacko A, Fabiano GA, Doctoroff GL, et al. Engaging fathers in effective parenting for preschool children using shared book reading: A randomized controlled trial. Journal of Clinical Child \& Adolescent Psychology. 2018 Jan 2;47(1):79-93.

25. Villar J, Fenandes M, Purwar M, et al. Neurodevelopmental milesto-

Grazie alle dottoresse Alessandra Sila e Valeria Balbinot del Centro per la Salute del Bambino per la collaorazione alla revisione del testo. nes and associated behaviours are similar among healthy children across diverse geographical locations. Nature communications. 2019;10:511 